\title{
Yield and composition of milk from Polish Holstein-Friesian and Jersey cows in particular months of the first lactations as dependent on milk protein polymorphism (short communication)
}

\begin{abstract}
The experiment was performed on 121 primiparous Polish Holstein-Friesian (PHF) cows and 36 primiparous Jersey (JER) cows. The highest decrease in casein yield was noted in JER cows at $7.36 \%$ on day 90 of lactation, $15.95 \%$ on day $150,26.78 \%$ on day $210,37.01 \%$ on day 270 and $39.67 \%$ on day 330 . In PHF cows this decrease was substantially lower, at $4.98 \%, 7.65 \%, 8.72 \%, 10.50 \%$ and $16.19 \%$ respectively. The casein content of milk from primiparous PHF cows ranged from 2.38\% (LGB - day 60) to 3.09\% (LGB BB - day $330)$, and it was found to be considerably lower in JER cows, varying between $2.35 \%$ (CSN3 BB - day 30$)$ and $3.61 \%$ (CSN3 BB - day 210). The highest casein yield was recorded in PHF cows with the following genotypes: LGB AA (day 60), CSN1S1 BB (day 60), CSN2 AB (day 270), CSN3 BB (day 150). In JER cows the highest casein yield was noted on day 90 of lactation with respect to the following genotypes: LGB BB, CSN1S1 BC, CSN2 AA and CSN3 BB. In PHF cows the greatest effect on whey protein yield was exerted by the following genotypes: LGB AA (day 90), CSN1S1 BC (day 60), CSN2 AA (day 90); the CSN3 BB genotype influenced whey protein yield in cattle of both breeds (day 90). In JER cows the highest daily yield of whey proteins was observed on day 90 of lactation, with regard to the following genotypes: LGB BB, CSN1S1 BC, CSN2 AB and CSN3 BB.
\end{abstract}

Keywords: Holstein-Friesian, Jersey, primiparous cows, month of lactation, casein, fat, whey proteins, milk protein polymorphism

\section{Zusammenfassung}

Titel der Arbeit: Leistung und Zusammensetzung von Milch bei Polnischen Holstein-Friesian und Jerseykühen in einzelnen Monaten der ersten Laktation abhängig vom Milcheiweißpolymorphismus (Kurzmitteilung)

Die Untersuchungen erfolgten an erstlaktierenden Kühen der Rassen Polnische Holstein Friesian (PHF) mit 121 und Jersey (JER) mit 36 Tieren. Das Ziel bestand in der Analyse der Milchleistung und -zusammensetzung in Abhängigkeit von den Milcheiweißpolymorphismen bei den einzelnen Laktationsmonaten. Im Laktationsverlauf zeigten die JER-Kühe die größere Abnahme des Kaseinertrages, erfasst an den Tagen 90, 150, 210, 230 bis 330 von 7,36; 15,9; 26,7; 37,01 und 39,67\%. Bei den PHF-Kühen war diese Abnahme geringer und betrug zu den gleichen Zeitpunkten nur 4,98; 7,65; 8,72; 10,50 und 16,19\%. Der Kaseingehalt bei den PHF-Kühen erstreckte sich von 2,38 B-Laktoglobulin (LGB) am 60. Tag bis 3,09 LGB BB am 330. Tag und lag beträchtlich niedriger als bei JER-Kühen mit 2,35\% (CSN3 BB) am 30. Tag und 3,61\% (CSN3 BB) am 210. Tag. Der höchste Kaseinertrag bei PHF Kühen fand sich bei den Genotypen LGB AA am 60. Tag, CSN1 BB am 270. Tag und CSN3 BB am 150. Tag. Dieser höchste Kasein- und Molkeneiweißertrag lag bei den JER-Kühen am 90. Laktationstag bei den Genotypen LGB BB, CSN1 S1 BC, CSN2 AA (Molkeneiweiß CSN AB) und CSN3 BB. Den größten Molkeneiweißertrag erzielten die PHF-Kühe der Genotypen LGB AA am 90. Tag, CSN1 S1 BC am 60. Tag und CSN2 AA am 90. Tag. In beiden Populationen lag dieser Ertrag bei den CSN3 BB Genotypen am 90. Tag am höchsten.

Schlüsselwörter: Holstein-Friesian, Jersey, Erstlaktation, Laktationmonate, Kasein, Fett, Molkeneiweiß, Milcheiweißpolymorphismus 


\section{Introduction}

It was demonstrated that two whey proteins, $\alpha$-lactalbumin (LALBA) and $\beta$-lactoglobulin (LGB), and four casein fractions, $\alpha$ s1 (CSN1S1), $\alpha$ s 2 (CSN1S2), $\beta$ (CSN2) and $\kappa(\mathrm{CSN} 3)$ exhibit polymorphism. Many authors who study milk protein polymorphism search for linkages between milk protein genes and the genes determining quantitative traits (JANN et al., 2002; GRAML and PIRCHNER, 2003). However, the above genes are located on numerous chromosomes, so such simple relationships cannot be found (ADACHI et al., 1996; KAMIŃSKI, 1996; KAMIŃSKI, 2000; KAMIŃSKI et al., 2002; JANN et al., 2002; CZERNIAWSKA-PIĄTKOWSKA et al., 2004). It has been reported that milk from cows with the CSN3 BB genotype has a higher percentage content of total protein, casein proteins and fat, is characterized by a shorter (by 10 to $30 \%$ ) coagulation time, provides a coagulate of greater firmness (by 20 to $100 \%$ ), retains more fat in the curd and ensures a higher cheese yield (JAKOB and PUCHAN, 1992; GERNAND and HARTUNG, 1997; KAMIŃSKI, 2000; JUSZCZAK et al., 2001). As regards the whey protein LGB, a higher milk yield is generally associated with the LGB AA genotype (OJALA et al., 1997; KAMIŃSKI et al., 2002), while a higher percentage content of fat and casein proteins - with the LGB BB genotype (WALAWSKI et al., 1994; LITWINCZUK et al., 1998). The selection of Black-and-White cattle oriented towards a higher milk yield results in an increased frequency of the CSN3 A allele and a decreased frequency of the B allele (KAMIŃSKI et al., 2002; CZERNIAWSKA-PIACTKOWSKA et al., 2004). Therefore, Black-and-White bulls with the AB genotype (containing the desired B allele) should be selected for breeding, and the relevant information should be placed in performance-tested bull catalogs. In Jersey cattle populations the frequency of the CSN1S1 B genotype is much higher, and milk has a high content of protein and fat (ALEANDRI et al., 1990; JANN et al., 2002). The objective of this study was to characterize the yield and composition of milk as dependent on polymorphic milk protein variants, as well as to determine the yield and content of casein and whey proteins in milk in particular months of lactation of primiparous Polish HolsteinFriesian and Jersey cows.

\section{Materials and Methods}

The experiment was performed during the years 2002-2006, on 121 primiparous Polish Holstein-Friesian (PHF) cows and 36 primiparous Jersey (JER) cows. The following milk performance traits were analyzed: milk yield, milk fat yield, milk protein yield, casein yield, dry matter content of milk, whey protein yield. Milk samples were collected individually from cows of both breeds on the following days during the first lactation ( \pm 5 days): 30, 60, 90, 150, 210, 270 and 330. In addition, at the beginning of lactation milk samples for the determination of milk protein genetic variants were taken directly from the teats of each cow, and poured into $1.5 \mathrm{ml}$ Eppendorf tubes. Those samples were used to determine the genetic variants of milk protein fractions by starch gel electrophoresis in the alkaline environment - according to the method developed by SCHMIDT (1964) and modified by Michalak (1969), and in the acid environment - as described by PETERSON and KOPFLER (1966). Four major proteins were obtained as a result of separation, i.e. $\alpha$ s1-casein (CSN1S1), $\beta$-lactoglobulin (LGB), $\kappa$-casein (CSN3) and $\beta$-casein (CSN2). 
The numerical data were verified using the following mathematical model:

$$
\mathrm{Y}_{\mathrm{ijk}}=\mu+\mathrm{A}_{\mathrm{i}}+\mathrm{B}_{\mathrm{j}}+\mathrm{C}_{\mathrm{k}}+\mathrm{E}_{\mathrm{ijk}}
$$

where: $Y_{i j k}$ - analyzed trait, $\mu$ - overall mean, $A_{i}-$ effect of the $i^{\text {th }}$ breed group $(i=1,2)$, $B_{j}$ - effect of the $j^{\text {th }}$ group of the investigated polymorphic protein system $(j=1, \ldots 4)$, $\mathrm{C}_{\mathrm{k}}$ - effect of the $\mathrm{k}^{\text {th }}$ genotype $(\mathrm{k}=1, \ldots 10), \mathrm{E}_{\mathrm{ijk}}$ - random error.

The results were verified statistically by analysis of variance and Tukey's test, using Statistica ver. 6.0 software (STATSOFT, 2003).

\section{Results}

In the case of the proteins LGB, CSN2 and CSN3, the AA and AB genotypes and the A gene occurred most frequently in the investigated population, regardless of breed. As regards the protein CSN1S1, almost all animals (120 PHF cows and 31 JER cows) had the BB genotype.

Table 1 presents the daily yields of milk and selected milk components in cattle of both breeds. In PHF cows the highest average yield of milk and milk components was noted in the second month of lactation (day 60), which was confirmed by statistically significant differences $(p \leq 0.01$ and $p \leq 0.05)$ between the mean values of the analyzed traits. In JER cows the highest productivity was recorded in the first and second month of lactation, and it remained at a high level until day 90. Average daily milk yield was lower in JER cows than in PHF cows. In the first and second month of lactation, JER cows produced 4 and $6 \mathrm{~kg}$ milk less than PHF cows, respectively, whereas in the last month of lactation (day 330) this difference reached $8 \mathrm{~kg}$.

Table 1

Daily yields of milk and milk components (Täglicher Milchertrag und Milchbestandteile)

\begin{tabular}{|c|c|c|c|c|c|c|c|c|}
\hline \multirow[t]{2}{*}{ Specification } & \multirow{2}{*}{$\begin{array}{l}\text { Statistical } \\
\text { measures }\end{array}$} & \multicolumn{7}{|c|}{ Lactation ( \pm 5 days $)$} \\
\hline & & 30 & 60 & 90 & 150 & 210 & 270 & 330 \\
\hline \multicolumn{9}{|c|}{ Polish Holstein-Friesian cows (PHF) } \\
\hline \multirow{2}{*}{ Milk [kg] } & LSM & $22^{\mathrm{ABa}}$ & $24^{\mathrm{CDE}}$ & $22^{\mathrm{DEFb}}$ & 20 & $18^{\mathrm{Eab}}$ & $17^{\mathrm{BDF}}$ & $16^{\mathrm{ACDE}}$ \\
\hline & $\mathrm{Sd}$ & 5.01 & 5.64 & 5.62 & 4.98 & 4.22 & 4.17 & 4.43 \\
\hline \multirow{2}{*}{ Fat $[\mathrm{g}]$} & LSM & $852^{\mathrm{ABa}}$ & $862^{\mathrm{CDE}}$ & $823^{\mathrm{DEFb}}$ & 802 & $794^{\mathrm{Eab}}$ & $779^{\mathrm{BDF}}$ & $765^{\mathrm{ACDE}}$ \\
\hline & $\mathrm{Sd}$ & 214.65 & 203.23 & 197.69 & 165.38 & 145.48 & 173.41 & 192.11 \\
\hline \multirow{2}{*}{ Total protein $[\mathrm{g}]$} & LSM & $667^{\mathrm{ad}}$ & $697^{\mathrm{Ae}}$ & $689^{c}$ & $662^{\mathrm{b}}$ & 635 & $617^{\mathrm{de}}$ & $601^{\text {Aabc }}$ \\
\hline & $\mathrm{Sd}$ & 169.04 & 186.15 & 183.42 & 164.99 & 140.00 & 149.33 & 148.33 \\
\hline \multirow{2}{*}{ Solids-non-fat [g] } & LSM & $1878^{\mathrm{Cc}}$ & $2028^{\mathrm{Aa}}$ & $1933^{\mathrm{Bb}}$ & 1754 & 1659 & $1577^{\mathrm{abc}}$ & $1474^{\mathrm{ABC}}$ \\
\hline & $\mathrm{Sd}$ & 450.88 & 514.24 & 499.09 & 436.76 & 380.44 & 384.33 & 375.25 \\
\hline \multirow{2}{*}{ Casein $[\mathrm{g}]$} & LSM & $543^{b}$ & $562^{\mathrm{ac}}$ & 534 & 519 & 513 & $505^{c}$ & $471^{\mathrm{ab}}$ \\
\hline & $\mathrm{Sd}$ & 134.12 & 150.88 & 139.38 & 136.80 & 116.21 & 127.04 & 131.22 \\
\hline \multirow{2}{*}{ Whey proteins $[\mathrm{g}]$} & LSM & 131 & $140^{\mathrm{b}}$ & $148^{\mathrm{c}}$ & $145^{\mathrm{a}}$ & 130 & $137^{\mathrm{A}}$ & $123^{\mathrm{abc}}$ \\
\hline & $\mathrm{Sd}$ & 52.05 & 64.60 & 59.73 & 52.01 & 41.67 & 49.02 & 40.11 \\
\hline \multicolumn{9}{|l|}{ Jersey cows (JER) } \\
\hline \multirow{2}{*}{ Milk [kg] } & LSM & $18^{\mathrm{ABa}}$ & $18^{\mathrm{CDE}}$ & $17^{\mathrm{DEFb}}$ & 13 & $11^{\mathrm{Eab}}$ & $9^{\mathrm{BDF}}$ & $8^{\mathrm{ACDE}}$ \\
\hline & $\mathrm{Sd}$ & 2.73 & 3.64 & 2.99 & 2.85 & 2.36 & 2.27 & 2.02 \\
\hline \multirow{2}{*}{ Fat $[\mathrm{g}]$} & LSM & $886^{\mathrm{Cb}}$ & $903^{\mathrm{Aa}}$ & $871^{\mathrm{Bc}}$ & 782 & 719 & $614^{\mathrm{abc}}$ & $568^{\mathrm{ABC}}$ \\
\hline & $\mathrm{Sd}$ & 229.65 & 174.23 & 129.06 & 159.78 & 148.55 & 141.90 & 122.40 \\
\hline \multirow{2}{*}{ Total protein $[\mathrm{g}]$} & LSM & $659^{\mathrm{Ac}}$ & $639^{\mathrm{Bb}}$ & 605 & $561^{\mathrm{a}}$ & 469 & $402^{\mathrm{bc}}$ & $369^{\mathrm{ABa}}$ \\
\hline & $\mathrm{Sd}$ & 96.01 & 131.43 & 124.68 & 123.33 & 110.76 & 94.23 & 84.05 \\
\hline \multirow{2}{*}{ Solids-non-fat [g] } & LSM & $1593^{\mathrm{BE}}$ & $1630^{\mathrm{AD}}$ & $1574^{\mathrm{CF}}$ & $1272^{\mathrm{a}}$ & 1023 & $874^{\mathrm{DEF}}$ & $810^{\mathrm{ABCa}}$ \\
\hline & $\mathrm{Sd}$ & 232.77 & 347.40 & 289.31 & 283.08 & 233.33 & 207.31 & 193.91 \\
\hline \multirow{2}{*}{ Casein [g] } & LSM & $489^{\text {ad }}$ & $479^{\text {be }}$ & $453^{\mathrm{cf}}$ & 411 & 358 & $308^{\mathrm{def}}$ & $295^{\mathrm{abc}}$ \\
\hline & $\mathrm{Sd}$ & 77.67 & 115.43 & 98.90 & 81.60 & 83.03 & 71.00 & 73.02 \\
\hline \multirow{2}{*}{ Whey proteins [g] } & LSM & $170^{\mathrm{Ab}}$ & $151^{\mathrm{Bc}}$ & $153^{\mathrm{ad}}$ & $150^{\mathrm{C}}$ & 121 & $100^{\text {bcd }}$ & $94^{\mathrm{ABCa}}$ \\
\hline & $\mathrm{Sd}$ & 49.55 & 46.04 & 50.28 & 50.22 & 30.76 & 33.21 & 26.76 \\
\hline
\end{tabular}

Means within traits, determined for particular lactation days, followed by the same letters differ significantly at: capital letters (p $\leq 0.01$ ) small letters $(\mathrm{p} \leq 0.05)$ 
The opposite tendency was observed only with respect to the daily yields of fat and whey proteins, which were higher in JER cows than in PHF cows during the first three months of lactation, and amounted to $886 \mathrm{~g}$ and $170 \mathrm{~g}$ (day 30), $903 \mathrm{~g}$ and $151 \mathrm{~g}$ (day $60), 871 \mathrm{~g}$ and $153 \mathrm{~g}$ (day 90), respectively. Considerable interbreed differences were found with regard to the yield of the most valuable milk protein - casein. Over the analyzed lactation the highest average casein yield was $562 \mathrm{~g}$ (PHF cows, day 60, $\mathrm{p} \leq 0.05)$ and $489 \mathrm{~g}(\mathrm{JER}$ cows, day $30, \mathrm{p} \leq 0.05)$. The highest decrease in casein yield (in comparison with the highest casein yield recorded on day 30) was noted in JER cows, at $7.36 \%$ on day 90 of lactation, $15.957 \%$ on day $150,26.78 \%$ on day 210 , $37.01 \%$ on day 270 and $39.67 \%$ on day 330 . In PHF cows this decrease was lower, at $4.98 \%, 7.65 \%, 8.72 \%, 10.50 \%$ and $16.19 \%$ respectively.

Table 2 shows dependencies between casein content and the genetic variants of milk proteins. In PHF cows the highest casein content of milk was recorded on day 330 of lactation.

Table 2

Daily casein yield (\%)(Täglicher Kaseinertrag [\%])

\begin{tabular}{|c|c|c|c|c|c|c|c|c|c|c|c|}
\hline \multirow{2}{*}{\multicolumn{2}{|c|}{ Specification }} & \multicolumn{3}{|c|}{ LGB } & \multicolumn{2}{|c|}{ CSN1S1 } & \multicolumn{2}{|c|}{ CSN2 } & \multicolumn{3}{|c|}{ CSN3 } \\
\hline & & AA & $\mathrm{AB}$ & $\mathrm{BB}$ & $\mathrm{BB}$ & $\mathrm{BC}$ & AA & $\mathrm{AB}$ & AA & $\mathrm{AB}$ & $\mathrm{BB}$ \\
\hline \multicolumn{12}{|c|}{ Polish Holstein-Friesian cows (PHF) } \\
\hline \multirow{2}{*}{30} & LSM & $2.50^{\mathrm{A}}$ & $2.47^{\mathrm{A}}$ & $2.45^{\mathrm{A}}$ & $2.47^{\mathrm{A}}$ & 2.50 & $2.47^{\mathrm{A}}$ & $2.56^{\mathrm{A}}$ & $2.47^{\mathrm{A}}$ & $2.49^{\mathrm{A}}$ & $2.42^{\mathrm{a}}$ \\
\hline & $\mathrm{Sd}$ & 0.21 & 0.26 & 0.25 & 0.25 & & 0.24 & 0.30 & 0.25 & 0.24 & 0.31 \\
\hline \multirow{2}{*}{60} & LSM & $2.38^{\mathrm{A}}$ & $2.38^{\mathrm{A}}$ & $2.38^{\mathrm{A}}$ & $2.38^{\mathrm{A}}$ & 2.21 & $2.38^{\mathrm{A}}$ & $2.39^{\mathrm{A}}$ & $2.40^{\mathrm{A}}$ & $2.34^{\mathrm{A}}$ & $2.46^{\mathrm{a}}$ \\
\hline & $\mathrm{Sd}$ & 0.22 & 0.28 & 0.23 & 0.25 & & 0.25 & 0.26 & 0.23 & 0.27 & 0.78 \\
\hline \multirow{2}{*}{90} & LSM & $2.43^{\mathrm{A}}$ & $2.43^{\mathrm{A}}$ & $2.40^{\mathrm{A}}$ & $2.42^{\mathrm{A}}$ & 2.38 & $2.42^{\mathrm{A}}$ & $2.57^{\mathrm{B}}$ & $2.43^{\mathrm{A}}$ & $2.40^{\mathrm{A}}$ & $2.40^{\mathrm{a}}$ \\
\hline & $\mathrm{Sd}$ & 0.19 & 0.23 & 0.16 & 0.21 & & 0.21 & 0.15 & 0.22 & 0.18 & 0.04 \\
\hline \multirow{2}{*}{150} & LSM & $2.71^{\mathrm{B}}$ & $2.66^{\mathrm{B}}$ & $2.69^{\mathrm{B}}$ & $2.68^{\mathrm{B}}$ & 2.80 & $2.67^{\mathrm{B}}$ & $2.85^{\mathrm{C}}$ & $2.65^{\mathrm{A}}$ & $2.73^{B}$ & $2.82^{\mathrm{b}}$ \\
\hline & $\mathrm{Sd}$ & 0.25 & 0.24 & 0.20 & 0.23 & & 0.23 & 0.22 & 0.23 & 0.23 & 0.15 \\
\hline \multirow{2}{*}{210} & LSM & $2.88^{\mathrm{B}}$ & $2.76^{\mathrm{B}}$ & $2.76^{\mathrm{B}}$ & $2.79^{\mathrm{B}}$ & 2.72 & $2.79^{\mathrm{B}}$ & $2.83^{\mathrm{C}}$ & $2.80^{\mathrm{B}}$ & $2.79^{\mathrm{B}}$ & $2.57^{\mathrm{a}}$ \\
\hline & $\mathrm{Sd}$ & 0.29 & 0.24 & 0.17 & 0.25 & & 0.25 & 0.19 & 0.25 & 0.25 & 0.07 \\
\hline \multirow{2}{*}{270} & LSM & $2.78^{\mathrm{B}}$ & $2.91^{\mathrm{C}}$ & $2.83^{\mathrm{B}}$ & $2.85^{\mathrm{B}}$ & 2.50 & $2.85^{\mathrm{B}}$ & $2.68^{A}$ & $2.84^{\mathrm{B}}$ & $2.94^{\mathrm{C}}$ & $2.52^{\mathrm{a}}$ \\
\hline & $\mathrm{Sd}$ & 0.22 & 0.30 & 0.23 & 0.26 & & 0.26 & 0.26 & 0.27 & 0.22 & 0.11 \\
\hline \multirow{2}{*}{330} & LSM & $3.00^{\mathrm{B}}$ & $3.03^{\mathrm{C}}$ & $3.09^{C}$ & $3.03^{\mathrm{C}}$ & 2.94 & $3.04^{\mathrm{C}}$ & $2.87^{\mathrm{C}}$ & $3.05^{\mathrm{C}}$ & $3.01^{\mathrm{C}}$ & $2.65^{\mathrm{b}}$ \\
\hline & $\mathrm{Sd}$ & 0.33 & 0.30 & 0.36 & 0.32 & & 0.32 & 0.31 & 0.31 & 0.33 & 0.35 \\
\hline \multicolumn{12}{|c|}{ Jersey cows (JER) } \\
\hline \multirow{2}{*}{30} & LSM & $2.47^{\mathrm{A}}$ & $2.53^{\mathrm{A}}$ & $2.69^{\mathrm{A}}$ & $2.55^{\mathrm{A}}$ & $2.54^{\mathrm{A}}$ & $2.54^{\mathrm{A}}$ & $2.55^{\mathrm{A}}$ & $2.52^{\mathrm{A}}$ & $2.60^{\mathrm{A}}$ & $2.35^{\mathrm{A}}$ \\
\hline & $\mathrm{Sd}$ & 0.14 & 0.22 & 0.23 & 0.22 & 0.18 & 0.22 & 0.17 & 0.19 & 0.23 & 0.15 \\
\hline \multirow{2}{*}{60} & LSM & $2.63^{\mathrm{A}}$ & $2.67^{\mathrm{A}}$ & $2.98^{\mathrm{B}}$ & $2.74^{\mathrm{B}}$ & $2.59^{\mathrm{A}}$ & $2.70^{\mathrm{B}}$ & $2.77^{\mathrm{B}}$ & $2.71^{\mathrm{B}}$ & $2.72^{\mathrm{A}}$ & $2.77^{\mathrm{B}}$ \\
\hline & $\mathrm{Sd}$ & 0.23 & 0.28 & 0.13 & 0.26 & 0.29 & 0.27 & 0.25 & 0.24 & 0.32 & 0.26 \\
\hline \multirow{2}{*}{90} & LSM & $2.84^{\mathrm{B}}$ & $2.93^{\mathrm{B}}$ & $3.16^{\mathrm{C}}$ & $2.96^{\mathrm{C}}$ & $2.79^{\mathrm{B}}$ & $2.91^{\mathrm{C}}$ & $3.01^{\mathrm{B}}$ & $2.89^{\mathrm{B}}$ & $2.97^{\mathrm{B}}$ & $3.12^{\mathrm{C}}$ \\
\hline & $\mathrm{Sd}$ & 0.25 & 0.29 & 0.21 & 0.26 & 0.36 & 0.28 & 0.24 & 0.25 & 0.30 & 0.37 \\
\hline \multirow{2}{*}{150} & LSM & $3.19^{C}$ & $3.12^{\mathrm{C}}$ & $3.18^{\mathrm{C}}$ & $3.18^{\mathrm{D}}$ & $3.03^{\mathrm{C}}$ & $3.14^{\mathrm{D}}$ & $3.25^{\mathrm{C}}$ & $3.20^{\mathrm{C}}$ & $3.06^{\mathrm{C}}$ & $3.33^{\mathrm{D}}$ \\
\hline & $\mathrm{Sd}$ & 0.23 & 0.20 & 0.21 & 0.19 & 0.28 & 0.18 & 0.30 & 0.20 & 0.18 & 0.26 \\
\hline \multirow{2}{*}{210} & LSM & $3.41^{\mathrm{D}}$ & $3.31^{\mathrm{D}}$ & $3.58^{\mathrm{D}}$ & $3.42^{\mathrm{E}}$ & $3.33^{\mathrm{D}}$ & $3.41^{\mathrm{E}}$ & $3.37^{\mathrm{C}}$ & $3.36^{\mathrm{C}}$ & $3.44^{\mathrm{D}}$ & $3.61^{\mathrm{E}}$ \\
\hline & $\mathrm{Sd}$ & 0.35 & 0.34 & 0.24 & 0.33 & 0.42 & 0.36 & 0.20 & 0.40 & 0.26 & 0.11 \\
\hline \multirow{2}{*}{270} & LSM & $3.33^{\mathrm{D}}$ & $3.48^{\mathrm{D}}$ & $3.56^{\mathrm{D}}$ & $3.45^{\mathrm{E}}$ & $3.34^{\mathrm{D}}$ & $3.44^{\mathrm{E}}$ & $3.40^{\mathrm{C}}$ & $3.47^{\mathrm{C}}$ & $3.51^{\mathrm{D}}$ & $3.31^{\mathrm{F}}$ \\
\hline & $\mathrm{Sd}$ & 0.22 & 0.35 & 0.22 & 0.30 & 0.19 & 0.30 & 0.21 & 0.24 & 0.32 & 0.31 \\
\hline \multirow{2}{*}{330} & LSM & $3.32^{\mathrm{D}}$ & $3.33^{\mathrm{D}}$ & $3.49^{\mathrm{D}}$ & $3.40^{\mathrm{E}}$ & $3.20^{\mathrm{D}}$ & $3.31^{\mathrm{E}}$ & $3.32^{\mathrm{D}}$ & $3.37^{\mathrm{C}}$ & $3.20^{\mathrm{D}}$ & $3.56^{\mathrm{G}}$ \\
\hline & $\mathrm{Sd}$ & 0.31 & 0.67 & 0.26 & 0.43 & 0.05 & 0.38 & 0.42 & 0.23 & 0.56 & 0.27 \\
\hline
\end{tabular}

Means determined for particular lactation days, followed by different letters in columns differ significantly at: capital letters ( $\leq \leq 0.01)$; small letters $(\mathrm{p} \leq 0.05)$

Differences between the above value and those determined in the other months were statistically significant at $p \leq 0.01$ and $p \leq 0.05$. In JER cows the highest casein content of milk was noted on day 210 and 270 of lactation $(p \leq 0.01)$. During the first lactation analyzed in the study the casein content of milk from PHF cows ranged from 2.38 
(LGB - day 60) to $3.09 \%$ (LGB BB - day 330), and it was found to be considerably lower in JER cows, varying between $2.35 \%$ (CSN3 BB - day 30 ) and $3.61 \%$ (CSN3 $\mathrm{BB}$ - day 210). In milk from cows of both breeds casein concentration increased with the progress of lactation. In PHF cows this increase started in the second month, while in JER cows - at the beginning of lactation. In the JER population a tendency to a higher casein content of milk was observed in cows with the following genotypes: LGB (BB>AA, AB), CSN1S1 (BB>BC), CSN2 (AB $>A A)$ and $C S N 3(B B>A B, A A)$.

Table 3 presents relationships between daily casein yield in particular phases of lactation and the genetic variants of milk proteins. Daily casein yield decreased with the progress of lactation, as confirmed by statistically significant differences at $p \leq 0.01$. The highest casein yield was recorded in PHF cows with the following genotypes: LGB AA (day 60), CSN1S1 BB (day 60), CSN2 AB (day 270), CSN3 BB (day 150). In JER cows the highest casein yield was noted on day 90 of lactation, with respect to the following genotypes: LGB BB, CSN1S1 BC, CSN2 AA and CSN3 BB.

Table 3

Daily casein yield (g) (Täglicher Milchertrag [g])

\begin{tabular}{|c|c|c|c|c|c|c|c|c|c|c|c|}
\hline \multirow{2}{*}{\multicolumn{2}{|c|}{ Specification }} & \multicolumn{3}{|c|}{ LGB } & \multicolumn{2}{|c|}{ CSN1S1 } & \multicolumn{2}{|c|}{ CSN2 } & \multicolumn{3}{|c|}{ CSN3 } \\
\hline & & AA & AB & $\mathrm{BB}$ & $\mathrm{BB}$ & $\mathrm{BC}$ & AA & $\mathrm{AB}$ & $\mathrm{AA}$ & $\mathrm{AB}$ & BB \\
\hline \multicolumn{12}{|c|}{ Polish Holstein-Friesian cows (PHF) } \\
\hline \multirow{2}{*}{30} & LSM & $589^{\mathrm{A}}$ & $527^{\mathrm{A}}$ & $526^{\mathrm{A}}$ & $543^{\mathrm{A}}$ & 538 & $545^{\mathrm{A}}$ & $484^{\mathrm{A}}$ & $555^{\mathrm{A}}$ & $512^{\mathrm{A}}$ & $575^{\mathrm{A}}$ \\
\hline & $\mathrm{Sd}$ & 173.04 & 115.43 & 117.76 & 135.23 & - & 134.09 & 156.20 & 137.56 & 128.29 & 76.03 \\
\hline \multirow{2}{*}{60} & LSM & $621^{\mathrm{B}}$ & $536^{\mathrm{A}}$ & $548^{\mathrm{B}}$ & $563^{\mathrm{B}}$ & 482 & $566^{\mathrm{B}}$ & $382^{\mathrm{B}}$ & $589^{\mathrm{B}}$ & $501^{\mathrm{A}}$ & $577^{\mathrm{A}}$ \\
\hline & $\mathrm{Sd}$ & 169.87 & 142.21 & 128.56 & 150.33 & - & 149.43 & 78.18 & 151.00 & 132.01 & 171.40 \\
\hline \multirow{2}{*}{90} & LSM & $591^{\mathrm{C}}$ & $529^{\mathrm{A}}$ & $475^{\mathrm{C}}$ & $543^{A}$ & 518 & $533^{\mathrm{A}}$ & $551^{\mathrm{C}}$ & $542^{\mathrm{A}}$ & $500^{\mathrm{A}}$ & $639^{\mathrm{B}}$ \\
\hline & $\mathrm{Sd}$ & 154.21 & 141.33 & 82.94 & 139.23 & - & 135.88 & 284.22 & 136.80 & 137.22 & 207.11 \\
\hline \multirow{2}{*}{150} & LSM & $585^{\mathrm{C}}$ & $492^{\mathrm{B}}$ & $491^{\mathrm{D}}$ & $521^{\mathrm{C}}$ & 280 & $517^{\mathrm{C}}$ & $565^{\mathrm{C}}$ & $518^{\mathrm{C}}$ & $496^{\mathrm{A}}$ & $773^{\mathrm{C}}$ \\
\hline & $\mathrm{Sd}$ & 184.27 & 109.89 & 79.59 & 134.33 & - & 122.98 & 373.48 & 121.32 & 129.00 & 304.03 \\
\hline \multirow{2}{*}{210} & LSM & $583^{\mathrm{C}}$ & $479^{\mathrm{B}}$ & $505^{\mathrm{D}}$ & $516^{\mathrm{C}}$ & $280^{\mathrm{b}}$ & $511^{\mathrm{C}}$ & $576^{\mathrm{C}}$ & $522^{\mathrm{C}}$ & $470^{\mathrm{B}}$ & $684^{\mathrm{D}}$ \\
\hline & $\mathrm{Sd}$ & 147.46 & 96.04 & 68.44 & 114.95 & - & 107.22 & 337.48 & 107.23 & 105.00 & 243.03 \\
\hline \multirow{2}{*}{270} & LSM & $543^{\mathrm{D}}$ & $502^{\mathrm{B}}$ & $440^{\mathrm{E}}$ & $507^{\mathrm{C}}$ & 323 & $501^{\mathrm{C}}$ & $607^{\mathrm{D}}$ & $502^{\mathrm{C}}$ & $488^{\mathrm{B}}$ & $632^{\mathrm{B}}$ \\
\hline & $\mathrm{Sd}$ & 115.55 & 133.49 & 112.24 & 126.05 & - & 121.33 & 323.05 & 127.36 & 110.78 & 176.05 \\
\hline \multirow{2}{*}{330} & LSM & $537^{\mathrm{D}}$ & $438^{\mathrm{C}}$ & $442^{\mathrm{F}}$ & $473^{\mathrm{D}}$ & 370 & $466^{\mathrm{D}}$ & $569^{\mathrm{C}}$ & $471^{\mathrm{D}}$ & $430^{\mathrm{C}}$ & $516^{\mathrm{D}}$ \\
\hline & $\mathrm{Sd}$ & 161.07 & 113.22 & 83.70 & 131.32 & - & 109.74 & 490.79 & 116.32 & 107.56 & 84.45 \\
\hline \multicolumn{12}{|c|}{ Jersey cows (JER) } \\
\hline \multirow{2}{*}{30} & LSM & $413^{\mathrm{A}}$ & $447^{\mathrm{A}}$ & $522^{\mathrm{A}}$ & $451^{\mathrm{A}}$ & $463^{\mathrm{A}}$ & $454^{\mathrm{A}}$ & $443^{\mathrm{A}}$ & $444^{\mathrm{A}}$ & $476^{\mathrm{A}}$ & $338^{\mathrm{A}}$ \\
\hline & $\mathrm{Sd}$ & 76.00 & 71.36 & 50.78 & 74.39 & 100.0 & 80.04 & 64.07 & 87.00 & 53.05 & 70.05 \\
\hline \multirow{2}{*}{60} & LSM & $453^{\mathrm{B}}$ & $471^{\mathrm{B}}$ & $518^{\mathrm{B}}$ & $474^{\mathrm{B}}$ & $508^{\mathrm{B}}$ & $487^{\mathrm{B}}$ & $453^{\mathrm{A}}$ & $464^{\mathrm{B}}$ & $516^{\mathrm{B}}$ & $414^{\mathrm{B}}$ \\
\hline & $\mathrm{Sd}$ & 102.60 & 130.75 & 96.43 & 115.04 & 129.0 & 123.05 & 88.08 & 109.45 & 124.23 & 96.60 \\
\hline \multirow{2}{*}{90} & LSM & $499^{\mathrm{C}}$ & $471^{\mathrm{B}}$ & $541^{\mathrm{A}}$ & $482^{\mathrm{B}}$ & $532^{\mathrm{C}}$ & $494^{\mathrm{B}}$ & $471^{\mathrm{A}}$ & $483^{\mathrm{B}}$ & $490^{\mathrm{B}}$ & $532^{\mathrm{C}}$ \\
\hline & $\mathrm{Sd}$ & 77.64 & 116.34 & 89.00 & 96.59 & 113.3 & 108.30 & 49.00 & 90.06 & 109.63 & 126.22 \\
\hline \multirow{2}{*}{150} & LSM & $432^{\mathrm{A}}$ & $387^{\mathrm{C}}$ & $417^{\mathrm{C}}$ & $407^{\mathrm{C}}$ & $437^{\mathrm{A}}$ & $423^{\mathrm{A}}$ & $368^{\mathrm{B}}$ & $409^{\mathrm{C}}$ & $433^{\mathrm{C}}$ & $339^{\mathrm{A}}$ \\
\hline & $\mathrm{Sd}$ & 74.03 & 85.00 & 87.57 & 85.12 & 50.18 & 80.63 & 72.92 & 86.78 & 64.66 & 97.99 \\
\hline \multirow{2}{*}{210} & LSM & $386^{\mathrm{D}}$ & $329^{\mathrm{D}}$ & $359^{\mathrm{D}}$ & $358^{\mathrm{D}}$ & $361^{\mathrm{D}}$ & $357^{\mathrm{C}}$ & $363^{\mathrm{B}}$ & $349^{\mathrm{D}}$ & $373^{\mathrm{D}}$ & $330^{\mathrm{A}}$ \\
\hline & $\mathrm{Sd}$ & 90.37 & 77.47 & 77.66 & 87.55 & 63.98 & 83.49 & 95.00 & 75.04 & 89.66 & 147.43 \\
\hline \multirow{2}{*}{270} & LSM & $344^{\mathrm{E}}$ & $273^{\mathrm{E}}$ & $292^{\mathrm{E}}$ & $305^{\mathrm{E}}$ & $321^{\mathrm{Ea}}$ & $300^{\mathrm{Da}}$ & $354^{\mathrm{B}}$ & $327^{\mathrm{D}}$ & $292^{\mathrm{E}}$ & $238^{\mathrm{D}}$ \\
\hline & $\mathrm{Sd}$ & 53.33 & 77.06 & 67.46 & 74.33 & 60.85 & 73.43 & 37.00 & 58.03 & 81.02 & 73.05 \\
\hline \multirow{2}{*}{330} & LSM & $339^{\mathrm{E}}$ & $260^{\mathrm{E}}$ & $235^{\mathrm{F}}$ & $280^{\mathrm{F}}$ & $353^{\mathrm{Eb}}$ & $279^{\mathrm{Db}}$ & $435^{\mathrm{A}}$ & $295^{\mathrm{E}}$ & $268^{\mathrm{E}}$ & $232^{\mathrm{D}}$ \\
\hline & $\mathrm{Sd}$ & 72.04 & 45.69 & 42.46 & 73.05 & 39.55 & 56.34 & 89.96 & 73.28 & 81.00 & 69.72 \\
\hline
\end{tabular}

Means determined for particular lactation days, followed by different letters in columns differ significantly at: capital letters ( $\leq \leq 0.01)$; small letters $(\mathrm{p} \leq 0.05)$

Table 4 presents dependencies between the daily yield of whey proteins in particular phases of lactation and the genetic variants of milk proteins. In PHF cows the greatest effect on whey protein yield was exerted by the following genotypes: LGB AA (day 90), CSN1S1 BC (day 60), CSN2 AA (day 90) and CSN3 BB (day 90). In JER cows 
the highest daily yield of whey proteins was observed on day 90 of lactation, with regard to the following genotypes: LGB BB, CSN1S1 BC, CSN2 AB and CSN3 BB. Until day 210 of lactation, PHF and JER cows were characterized by a comparable average yield of whey proteins, which decreased in the JER population towards the end of lactation, i.e. on day 270 and 330.

\section{Discussion}

OJALA et al. (1997) reported similar genotype frequencies for the Holstein-Friesian breed, but somewhat different for the Jersey breed, i.e. 0.07 (LGB AA), 0.58 (LGB $\mathrm{AB}$ ) and 0.35 (LGB BB). JANN et al. (2002) determined the frequency of the CSN1S1 $\mathrm{B}$ allele in the Jersey population at 0.63 , compared to 0.93 in the present study. According to WALAWSKI et al. (1994), the CSN3 B gene - associated with a higher protein content of milk and higher processing suitability of milk in respect of cheese production - occurs with a higher frequency in JER cattle. The above authors demonstrated that regardless of lactation and sampling time the highest casein content was observed in cows with the LGB (AA>AB.BB) and CSN3 (AB>AA.BB) genotypes.

Table 4

Daily yield of whey proteins (g) (Täglicher Molkeneiweißertrag [g])

\begin{tabular}{|c|c|c|c|c|c|c|c|c|c|c|c|}
\hline \multirow{2}{*}{\multicolumn{2}{|c|}{ Specification }} & \multicolumn{3}{|c|}{ LGB } & \multicolumn{2}{|c|}{ CSN1S1 } & \multicolumn{2}{|c|}{ CSN2 } & \multicolumn{3}{|c|}{ CSN3 } \\
\hline & & AA & $\mathrm{AB}$ & $\mathrm{BB}$ & $\mathrm{BB}$ & $\mathrm{BC}$ & AA & $\mathrm{AB}$ & AA & $\mathrm{AB}$ & $\mathrm{BB}$ \\
\hline \multicolumn{12}{|c|}{ Polish Holstein-Friesian cows (PHF) } \\
\hline \multirow{2}{*}{30} & LSM & $134^{\mathrm{A}}$ & $136^{\mathrm{A}}$ & $120^{\mathrm{A}}$ & $132^{\mathrm{A}}$ & 110 & $133^{\mathrm{A}}$ & $92^{\mathrm{A}}$ & $137^{\mathrm{a}}$ & $116^{\mathrm{a}}$ & $162^{\mathrm{a}}$ \\
\hline & $\mathrm{Sd}$ & 43 & 61 & 38 & 52 & - & 52 & 07 & 56 & 37 & 17 \\
\hline \multirow{2}{*}{60} & LSM & $164^{\mathrm{B}}$ & $127^{\mathrm{A}}$ & $142^{\mathrm{B}}$ & $140^{\mathrm{B}}$ & 161 & $141^{\mathrm{A}}$ & $96^{\mathrm{A}}$ & $148^{\mathrm{a}}$ & $119^{\mathrm{a}}$ & $189^{b}$ \\
\hline & $\mathrm{Sd}$ & 67 & 47 & 87 & 64 & - & 64 & 18 & 71 & 36 & 81 \\
\hline \multirow{2}{*}{90} & LSM & $172^{\mathrm{B}}$ & $154^{\mathrm{B}}$ & $129^{\mathrm{A}}$ & $153^{\mathrm{B}}$ & 159 & $154^{\mathrm{B}}$ & $123^{\mathrm{B}}$ & $155^{\mathrm{b}}$ & $144^{\mathrm{b}}$ & $190^{\mathrm{b}}$ \\
\hline & $\mathrm{Sd}$ & 63 & 62 & 34 & 59 & - & 59 & 61 & 63 & 51 & 11 \\
\hline \multirow{2}{*}{150} & LSM & $163^{\mathrm{B}}$ & $138^{\mathrm{A}}$ & $139^{\mathrm{B}}$ & $145^{\mathrm{B}}$ & 105 & $146^{\mathrm{A}}$ & $112^{\mathrm{A}}$ & $152^{\mathrm{b}}$ & $127^{\mathrm{a}}$ & $166^{\mathrm{a}}$ \\
\hline & $\mathrm{Sd}$ & 55 & 51 & 47 & 52 & - & 53 & 13 & 52 & 49 & 45 \\
\hline \multirow{2}{*}{210} & LSM & $142^{\mathrm{A}}$ & $122^{\mathrm{A}}$ & $133^{\mathrm{B}}$ & $130^{\mathrm{C}}$ & 81 & $130^{\mathrm{A}}$ & $143^{C}$ & $130^{\mathrm{a}}$ & $121^{\mathrm{a}}$ & $113^{b}$ \\
\hline & $\mathrm{Sd}$ & 50 & 38 & 29 & 41 & - & 39 & 93 & 39 & 38 & 33 \\
\hline \multirow{2}{*}{270} & LSM & $160^{\mathrm{B}}$ & $122^{\mathrm{A}}$ & $121^{\mathrm{A}}$ & $136^{\mathrm{A}}$ & 95 & $134^{\mathrm{A}}$ & $198^{\mathrm{D}}$ & $137^{\mathrm{a}}$ & $120^{\mathrm{a}}$ & $100^{\mathrm{b}}$ \\
\hline & $\mathrm{Sd}$ & 51 & 44 & 37 & 49 & - & 46 & 117 & 43 & 54 & 71 \\
\hline \multirow{2}{*}{330} & LSM & $143^{\mathrm{A}}$ & $109^{\mathrm{C}}$ & $121^{\mathrm{A}}$ & $123^{\mathrm{A}}$ & 102 & $122^{\mathrm{C}}$ & $141^{\mathrm{C}}$ & $123^{c}$ & $113^{\mathrm{a}}$ & $116^{\mathrm{b}}$ \\
\hline & $\mathrm{Sd}$ & 50 & 34 & 28 & 41 & - & 38 & 106 & 40 & 32 & 87 \\
\hline \multicolumn{12}{|c|}{ Jersey cows (JER) } \\
\hline \multirow{2}{*}{30} & LSM & $152^{\mathrm{A}}$ & $134^{\mathrm{A}}$ & $176^{\mathrm{A}}$ & $149^{\mathrm{A}}$ & $145^{\mathrm{A}}$ & $146^{\mathrm{A}}$ & $162^{\mathrm{A}}$ & $156^{\mathrm{A}}$ & $143^{\mathrm{A}}$ & $89^{\mathrm{A}}$ \\
\hline & $\mathrm{Sd}$ & 61 & 41 & 44 & 50 & 51 & 46 & 77 & 53 & 43 & 48 \\
\hline \multirow{2}{*}{60} & LSM & $147^{\mathrm{A}}$ & $145^{\mathrm{A}}$ & $171^{\mathrm{A}}$ & $153^{\mathrm{A}}$ & $142^{\mathrm{A}}$ & $152^{\mathrm{A}}$ & $150^{\mathrm{A}}$ & $152^{\mathrm{A}}$ & $156^{\mathrm{A}}$ & $128^{\mathrm{B}}$ \\
\hline & $\mathrm{Sd}$ & 43 & 57 & 14 & 49 & 18 & 45 & 52 & 45 & 49 & 45 \\
\hline \multirow{2}{*}{90} & LSM & $180^{\mathrm{B}}$ & $154^{\mathrm{A}}$ & $191^{\mathrm{B}}$ & $169^{\mathrm{A}}$ & $173^{\mathrm{B}}$ & $169^{\mathrm{A}}$ & $172^{\mathrm{A}}$ & $175^{\mathrm{B}}$ & $157^{\mathrm{A}}$ & $214^{\mathrm{C}}$ \\
\hline & $\mathrm{Sd}$ & 46 & 55 & 40 & 52 & 47 & 55 & 34 & 51 & 47 & 63 \\
\hline \multirow{2}{*}{150} & LSM & $141^{\mathrm{A}}$ & $144^{\mathrm{A}}$ & $182^{\mathrm{B}}$ & $149^{\mathrm{A}}$ & $154^{\mathrm{A}}$ & $159^{\mathrm{A}}$ & $115^{\mathrm{B}}$ & $135^{\mathrm{C}}$ & $177^{\mathrm{B}}$ & $126^{\mathrm{B}}$ \\
\hline & $\mathrm{Sd}$ & 43 & 52 & 56 & 54 & 19 & 51 & 28 & 46 & 46 & 53 \\
\hline \multirow{2}{*}{210} & LSM & $123^{\mathrm{C}}$ & $117^{\mathrm{B}}$ & $124^{\mathrm{C}}$ & $122^{\mathrm{B}}$ & $115^{\mathrm{C}}$ & $120^{\mathrm{B}}$ & $125^{\mathrm{B}}$ & $116^{\mathrm{C}}$ & $127^{\mathrm{B}}$ & $120^{\mathrm{B}}$ \\
\hline & $\mathrm{Sd}$ & 38 & 23 & 32 & 31 & 26 & 29 & 39 & 31 & 31 & 37 \\
\hline \multirow{2}{*}{270} & LSM & $113^{C}$ & $83^{C}$ & $104^{C}$ & $97^{\mathrm{C}}$ & $114^{\mathrm{C}}$ & $95^{\mathrm{C}}$ & $132^{\mathrm{B}}$ & $105^{\mathrm{C}}$ & $96^{\mathrm{C}}$ & $73^{\mathrm{A}}$ \\
\hline & $\mathrm{Sd}$ & 30 & 28 & 38 & 35 & 15 & 32 & 15 & 3 & 34 & 22 \\
\hline \multirow{2}{*}{330} & LSM & $98^{\mathrm{C}}$ & $97^{\mathrm{C}}$ & $79^{\mathrm{D}}$ & $95^{\mathrm{C}}$ & $92^{\mathrm{C}}$ & $92^{\mathrm{C}}$ & $109^{\mathrm{B}}$ & $94^{\mathrm{C}}$ & $93^{C}$ & $86^{\mathrm{A}}$ \\
\hline & $\mathrm{Sd}$ & 35 & 17 & 03 & 24 & 45 & 27 & 21 & 26 & 10 & 18 \\
\hline
\end{tabular}

Means followed by the same letters in columns differ significantly at: capital letters $(\mathrm{p} \leq 0.01)$; small letters $(\mathrm{p} \leq 0.05)$ 
Comparable results were reported by BIELAK et al., (1991). Those authors distinguished three stages of changes in total protein and casein content: the first three months of lactation, when the concentrations of these components were at the lowest level (3.01-3.10\% and 2.35-2.42\% respectively) months 4 to 8 , when these concentrations were much higher (3.21-3.49\% and 2.53-2.75\%) and the last two months of lactation, when the content of both components was the highest (3.61-3.66 \% and 2.85-2.91\%). JUSZCZAK et al. (2001) confirmed a statistically significant $(p \leq 0.05)$ effect of the LGB BB and CSN3 AA genotypes on an increase in the fat content of milk from Black-and-White cows, which is consistent with the results of the present study. DYBUS and GRZESIAK (2006) also reported a higher yield of milk and fat as well as a higher fat content in Black-and-White cows with the AA genotype. The results obtained by DYBUS et al. (2005) with respect to Jersey cattle correspond to the present findings and indicate that primiparous cows with the CSN2 AA genotype are marked by a lower yield and content of milk fat and milk protein. CZERNIAWSKAPIACTKOWSKA et al. (2002) demonstrated a higher yield of milk, fat and protein in CSN3 BB cows, which also agrees with the data reported here. According to ALEANDRI et al. (1990), CSN3 has a beneficial influence on the yield and content of milk components (primarily protein).

\section{Acknowledgement}

This work was supported within the framework of statutory project of the Department of Cattle Breeding and Milk Quality Evaluation, UWM in Olsztyn no. 0106.0801 and by funds allocated to scientific research in 2006-2009 within the framework of grant of Ministry of Scientific Research and Information Technology no. N31105631/0237.

\section{References}

ADACHI, T.; AHN, J-Y.; YAMAMOTO, K.; AOKI, N.; NAKAMURA, R.; MATSUDA, T.: Characterisation of the bovine k-casein gene promoter. Biosci. Biotechnol. Biochem. 60 (1996), 19371940

ALEANDRI, R.; BUTTAZONI, L.G.; SCHNEIDER, J.C.; CAROLI, A.; DAVOLI, R.:

The effects of milk protein polymorphisms on milk components and cheese-producing ability. J. Dairy Sci. 73 (1990), 241-255

BIELAK, F.; WAWRZYŃCZAK, S.; GWOŹDZIEWICZ, A.:

Changes in the content of and correlations between milk components in successive months and phases of lactation in Lowland Black-and-Whitec cows. Rocz. Nauk. Zoot. t. 18 (1991), 87-99 [in Polish]

CZERNIAWSKA-PIAॄTKOWSKA, E.; KAMIENIECKI, H.; PILARCZYK, R.; RZEWUCKA, E.:

A comparison of protein polymorphisms in milk produced by two dairy farms in West Pomerania. Arch. Tierz. 47 (2004), 155-163

CZERNIAWSKA-PIĄTKOWSKA, E.; KAMIENIECKI, H.; MIKULA, A.:

Milk protein polymorphism versus milk productivity in a herd of cattle. Acta Scientiarum Polonorum. Zootechnika 1 (2002), 17-26 [in Polish]

CZERNIAWSKA-PIĄTKOWSKA, E.; ZYCH, S.; MUSZYŃSKA, M.:

Association of genetic variants of bovine prolactin with milk production traits of Black-and-White and Jersey cattle. Arch. Tierz. 48 (2005), 149-156

DYBUS, A.; GRZESIAK, W.:

GHRH/HaeIII gene polymorphism and its associations with milk production traits in Polish Black-andWhite cattle. Arch.Tierz. 49 (2006), 434-438

GERNAND, E.; HARTUNG, H.:

Untersuchungen zu Einflußgrößen auf Zusammensetzung und Käsereitauglichkeit von Rohmilch einzelner Kühe. 2. Mitt.: Untersuchungen zur Variation der Milchgerinnung und deren Ursachen an Einzelmilchproben. Arch. Tierz. 40 (1997), 225-238 
GRAML, R.; PIRCHNER, F.:

Effects of milk protein loci on content of their proteins. Arch. Tierz. 46 (2003), 331-340

JAKOB, E.; PUCHAN, Z.

Technological properties of milk as influenced by genetic polymorphism of milk proteins - a review. Intern. Dairy J. 2 (1992), 157-178

JANN, O.; PRINZENBERG, E.M.; BRANDT, H.; WILIAMS, J.L.; AJMONE-MARSAN, P.; ZARAGOZA, P.;

ÖZBEYAZ C.; ERHARDT, G.: Interagenic haplotypes At the bovine CSN1S1 locus. Arch. Tierz. 45 (2002), 13-21

JUSZCZAK, J.; ERHARDT, G.; KUCZAJ, M.; ZIEMIŃSKI, R.; PANICKE, L.:

Relations between genetic variants of kappa-casein and beta-globulin and performance of Black and White Polish Red cattle. Arch. Tierz. 44 (2001), 239-249

KAMIŃSKI, S.:

Associations between polymorphism within regulatory and coding fragment of bovine kappa-casein gene and milk performance traits. J. Anim. Feed Sci. 9 (2000), 435-446

KAMIŃSKI, S.:

Bovine kappa-casein (CASK) gene - molecular nature and application in dairy cattle breeding. J. Appl. Gen. 37 (1996), 179-196

KAMIŃSKI, S.; RYMKIEWICZ-SCHYMCZYK, J.; WÓJCIK, E.: RUŚĆ, A.:

Associations between bovine milk protein genotypes and haplotypes and the breeding value of Polish Black-and-White bulls. J. Anim. Feed Sci. 11 (2002), 205-221

LITWIŃCZUK, A.; LITWIŃCZUK, Z.; BARŁOWSKA, J.; FLOREK, M.:

Productivity and chemical composition of milk with particular reference to protein and its fractions in Black-and-White cows, Jersey cows, and cows with various proportion of HF genes. Mater. Konf. Nauk. Wrocław 25-26.06.1998, zesz. 331 (1998), 149-155 [in Polish]

MICHALAK, W.B.:

Application of starch gel electrophoresis for simultaneous determination of phenotypes of four main milk proteins. Part I. Biul. Z.H.D.Z. PAN 15 (1969), 75-88 [in Polish]

OJALA, M.; FAMULA, T.R.; MEDRANO, J.F.:

Effects of milk protein genotypes on the variation for milk production traits of Holstein and Jersey cows in California. J. Dairy Sci. 80 (1997), 1776-1785

PETERSON, R. F.; KOPFLER, F. C.:

Detection of new types of caseins by polyacrylamide gel electrophoresis at acid $\mathrm{pH}$ : a proposed nomenclature., Bioch. and Biophys. Research Communications, t. 22 (1966), 368

PEDERSEN, J.:

Selection to increase frequency of kappa-casein variant B in dairy cattle. J. Anim. Breed. Gen. 108 (1991), 434-445

SCHMIDT, D.C.:

Starch gel electrophoresis of kappa-casein. Biochim. Biophys. Acta 90 (1964), 411-415

STATSOFT:

Statistica. Data analysis software systems. Ver. 6.0 (2003)

http://www.statsoft.pl/base.html\#anovamanova

WALAWSKI, K.; SOWIŃSKI, G.; CZARNIK, U.; ZABOLEWICZ, T.:

Beta-lactoglobulin and kappa-casein polymorphism in relation to production traits and technological properties of milk in the herd of Polish Black-and-White cows. Genet. Pol. 35 (1994), 93-108

Received: 2007-02-21

Accepted: 2008-04-10

Authors:

Dr. inż. JAN MICIŃSKI*

Dr. MARIUSZ JASTRZĘBSKI

Prof. Dr. habil. JANUSZ KLUPCZYŃSKI

University of Warmia and Mazury in Olsztyn (UWM)

Department of Cattle Breeding and Milk Quality Evaluation

Oczapowskiego 5/150

10-957 Olsztyn

Poland

*Corresponding author

email: micinski@uwm.edu.pl 